\title{
Highly linear dual parallel Mach-Zehnder modulator incorporating MMI couplers
}

\author{
B. Mao \\ bmmao@stu.xidian.edu.cn \\ P. Yue \\ pengy@xidian.edu.cn \\ F. Hou
}

Z. Liu
State Key Laboratory of Integrated Services Networks, Xidian University, Xi'an, China

State Key Laboratory of Integrated Services Networks, Xidian University, Xi'an, China

State Key Laboratory of Integrated Services Networks, Xidian University, Xi'an, China

State Key Laboratory of Integrated Services Networks, Xidian University, Xi'an, China

The strict requirements on splitting ratios of optical power and radio-frequency (RF) voltage affect the performance of the Dual Parallel Mach-Zehnder modulator (DPMZM). In this paper, a modified DPMZM with three Multimode Interference (MMI) couplers (MMI-DPMZM) is proposed. The three MMI couplers, comprised of a reconfigurable one and two $3 \mathrm{~dB}$ ones, function as optical splitters. The theoretical analysis shows that the structure can prohibit the third-order intermodulation distortion (IMD3) from the third order term and fifth order term of the transfer function's Taylor series, leading to the peak Spurious-Free Dynamic Range (SFDR) as high as 104.12 dB. The adjustment of the tunable MMI coupler ensures the SFDR is kept above $104.00 \mathrm{~dB}$ even the radio frequency (RF) signal's voltage splitting ratio deviates from its optimum value. It is also demonstrated that the SFDR can reach $104.10 \mathrm{~dB}$ with the precision and uniformity of MMI couplers considered.

[DOI: http://dx.doi.org/10.2971/jeos.2015.15004]

Keywords: DPMZM, MMI coupler, dynamic range, deviation

\section{INTRODUCTION}

An increasing number of mobile Internet devices offering people various broadband wireless multimedia services have been driving the communications technology to make continuous progress. To meet the urgent demand on next-generation access networks for a large bandwidth and high mobility, the techniques of Radio over Fibre (RoF) and Radio over Free Space Optics (RoFSO) are proposed, which have been considered as the integrations of optical communications and wireless communications [1, 2]. The RoF and RoFSO systems have shown their advantages and high perspectives when integrated with modern sophisticated technologies, such as wavelength division multiplexing (WDM) systems [3], the radio access network of LTE (Long Term Evolution) [4]. Recently, several standards have been released to promote the global use of $60 \mathrm{GHz}$ wireless technology: wireless-HD [5] and WiGiG [6] for wireless local area networks (WLANs). RoF and RoFSO systems operating at $60-\mathrm{GHz}$ millimeter-wave (mmwave) will be an effective technology to increase the network capacity of these systems for the huge bandwidth over $7 \mathrm{GHz}$ unlicensed mm-wave band with spectral availability to achieve multi-gigabit data rate with very low power consumption [5].

In RoF and RoFSO systems, electrooptic modulators are one of the key components to ensure the high performance [7]. An electrooptic modulator with excellent linearity and high tolerance will be crucial for optimising the performance of these systems by improving the modulation efficiency. Researchers have put forward numerous structures to reduce the nonlinear distortion limiting the dynamic range in analog applications [8]-[10]. An important type among them is the Dual Parallel Mach-Zehnder modulator (DPMZM) which became a live topic after it was proposed $[8,11]$. Many researchers have applied it into the RoF and RoFSO links to fulfill different functions, such as single sideband (SSB) modulation [12], quadrature amplitude modulation (QAM) [13], optical mmwave signal generation [14]. In these systems, the linearity of the modulator is one of the significant factors to affect the Spurious Free Dynamic Range (SFDR) which is an important measure to evaluate these systems. When DPMZM works at its optimum operating point, the reduction of the intermodulation distortion (IMD) is more than $20 \mathrm{~dB}$, resulting in a high SFDR value. It can maintain its ultra linearity in a wide radio frequency (RF) range, which is very important for RoF and RoFSO links [11].

In DPMZM, the suppression of IMD is critically dependent on the values of the RF voltage splitting ratio and the optical power splitting ratio. In most papers, the optical power splitting ratio is chosen to be the cubic of the RF voltage splitting ratio, resulting in suppression of most of the third order intermodulation distortion (IMD3) which predominates in the IMD $[8,13]$. However, more IMD3 can be prohibited if the optical power splitting ratio is slightly off the cubic of the RF 
voltage splitting ratio, leading to further improvement of linearity [9]. The optimum operating condition requires that the optical power splitting ratio matches the RF voltage splitting ratio precisely. Otherwise, the linearity seriously deteriorates even if the values of two ratios are slightly unmatched. For the conventional DPMZM with Y splitters splitting the input light, it is necessary to implement an active feedback circuit driven by the IMD to control the optical power splitting ratio for the optimum performance. On the other hand, the feedback circuit may be complex and difficult to control due to the high precision requirement of the optical power splitting ratio [9].

Recently, the Multimode Interference (MMI) couplers attract a lot of attention because they can provide precise splitting with very low loss, large optical bandwidth and high insensitivity to fabrication tolerances as optical splitters [15]-[17]. They can be easily integrated with other devices owing to their compact sizes. Moreover, the reconfigurable types among them can switch the light between the output waveguides through the adjustment of the control circuits $[18,19]$. Such couplers are very useful when integrated within interferometric devices where the splitting ratios are crucial for the performance of these devices.

In this paper, we firstly present the theoretical model of a modified DPMZM with three Y-splitters replaced by three MMI couplers (MMI-DPMZM) consisting of a $1 \times 2$ tunable MMI coupler and two 1x2 $3 \mathrm{~dB}$ asymmetrical MMI couplers. The detailed analysis proves that the application of MMI couplers helps the modulator obtain a large SFDR over a wide operating range. Even if the RF voltage splitting ratio deviates from its optimal value, the deterioration of SFDR can be compensated by the adjustment of the tunable MMI coupler. The structure is also tolerant to manufacturing errors of MMI couplers and the SFDR decreases slightly when manufacturing errors of modern MMI couplers are considered.

\section{DESIGN}

The MMI couplers are the key parts in the novel structure. Figure 1(a) gives the structure of the $1 \times 2$ reconfigurable MMI coupler [18]. It consists of three parts: the input waveguide, the multimode section and the output waveguides. Four areas in the multimode section are covered by electrodes on which voltages of equal amplitudes and different polarities are applied. Through adjusting the four voltages synchronously, we can alter the refractive indices of these regions, leading to a modified phase relation of the self-images formed in these areas. Then the light between different waveguides can be switched [19]. If the applied voltages are zero, the device operates as a symmetrical $3 \mathrm{~dB}$ coupler. We assume that the power ratio of the output waveguides is $A: 1$. The value of $A$ can be changed through the adjustment of the voltages placed on the electrodes. The powers of the input light and output light can be given as follows:

$$
\left[\begin{array}{l}
P_{\text {out }, 1} \\
P_{\text {out }, 2}
\end{array}\right]=\left[\begin{array}{c}
\frac{A}{A+1} \\
\frac{1}{A+1}
\end{array}\right] P_{\text {in }}
$$

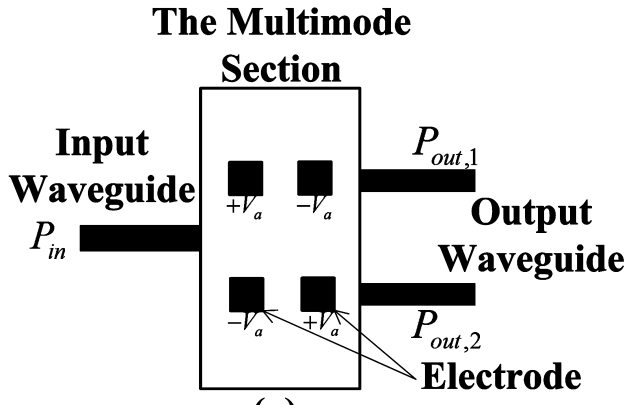

(a)

The Multimode

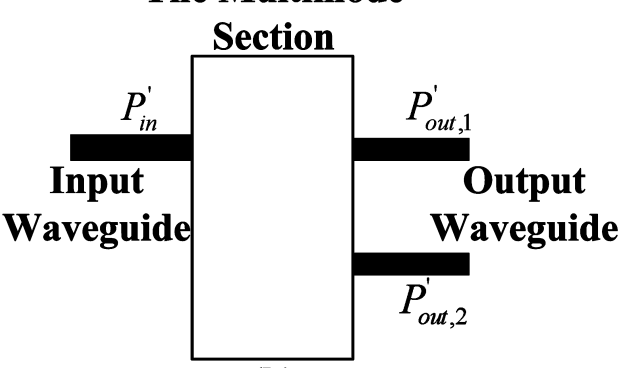

(b)

FIG. 1 (a) Structure of the reconfigurable MMI coupler, (b) Structure of the asymmetrical MMI coupler

where $P_{\text {in }}$ represents the power of the input light. $P_{\text {out }, 1}\left(P_{\text {out }, 2}\right)$ represents the power of the light in the upper (lower) output waveguide. Furthermore, the MMI coupler also provides relative phases between the output waveguides and the values of the relative phases change as the voltages on the electrodes.

As shown in Figure 1(b), the structure of the asymmetrical $3 \mathrm{~dB}$ MMI coupler is similar to that of the tunable MMI coupler while there is no electrode in the multimode section [20] and the position of input waveguide is different. The light is input at $1 / 3$ of the MMI width and split into two paths in equal powers with relative phases of $\{\pi / 2,0\}$ [20]. If the light is input at $2 / 3$ of the MMI width, the relative phases are $\{0, \pi / 2\}$. We express the relationship between the powers of output light and input light as:

$$
\left[\begin{array}{l}
P_{\text {out } 1}^{\prime} \\
P_{\text {out }, 2}^{\prime}
\end{array}\right]=\left[\begin{array}{c}
\frac{1}{2} \\
\frac{1}{2}
\end{array}\right] P_{\text {in }}^{\prime}
$$

where $P_{\text {in }}^{\prime}$ represents the power of the input light. $P_{\text {out }, 1}^{\prime}$ and $P_{\text {out }, 2}^{\prime}$ are the powers of light in the two output waveguides.

Figure 2 gives the structure of the proposed modulator. We use a tunable MMI coupler $\left(\mathrm{MMI}_{a}\right)$ and two asymmetrical $3 \mathrm{~dB}$ MMI couplers $\left(\mathrm{MMI}_{b}\right.$ and $\left.\mathrm{MMI}_{\mathcal{c}}\right)$ to substitute for the $\mathrm{Y}$ splitters. The input light is split by the reconfigurable MMI coupler and the power ratio of the output light is $A: 1$ as mentioned above. The value of $A$ can be adjusted if we change the amplitude of the voltages, $V_{a}$. The output light of $\mathrm{MMI}_{a}$ penetrates $\mathrm{MMI}_{b}$ and $\mathrm{MMI}_{c}$ respectively. $\mathrm{MMI}_{b}$ and $\mathrm{MMI}_{c}$ are similar except their input positions and offer different relative phases, which is to ensure the distortions in $\mathrm{MZM}_{b}$ and $\mathrm{MZM}_{c}$ have opposite phases and can cancel out each other in the output path of $\mathrm{MZM}_{a}$ [9]. The relative phases offered by $\mathrm{MMI}_{b}$ and $\mathrm{MMI}_{\mathcal{c}}$ are $\{0, \pi / 2\}$ and $\{\pi / 2,0\}$ respectively, helping $\mathrm{MZM}_{b}$ and $\mathrm{MZM}_{\mathcal{C}}$ maintain quadrature between their arms. There- 


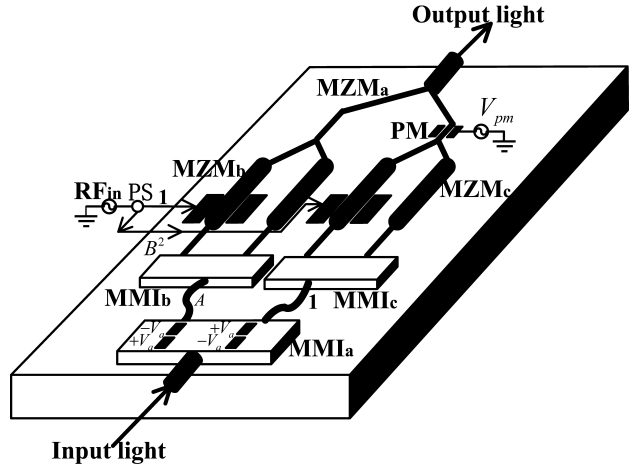

FIG. 2 Structure of MMI-DPMZM.

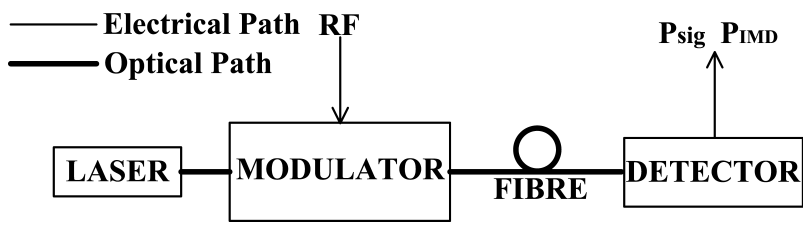

FIG. 3 Schematic Diagram of the model optical link.

fore, the DC bias circuits on the arms of $\mathrm{MZM}_{b}$ and $\mathrm{MZM}_{\mathcal{C}}$ can be omitted. When traveling through the arms of $\mathrm{MZM}_{b}$ and $\mathrm{MZM}_{c}$, the light is modulated by the RF signals split in a power ratio of $1: B^{2}$. We can take $B$ as the voltage ratio to simplify the calculation. The output light of $\mathrm{MZM}_{b}$ and $\mathrm{MZM}_{c}$ combines in the Y-combiner. A phase modulator (PM) is implemented on the output path of $\mathrm{MZM}_{c}$ to maintain quadrature between the two arms of $\mathrm{MZM}_{a}$ for the reason that the relative phases offered by $\mathrm{MMI}_{a}$ may be changed when $\mathrm{MMI}_{a}$ is adjusted [9]. Using Eqs. (1) and (2) and the transfer function of MZM [10], we can get the transfer function of MMI-DPMZM as follows:

$$
T=\frac{1}{2}\left[1+\frac{A}{A+1} \sin \left(\frac{\pi V}{V_{\pi}}\right)-\frac{1}{A+1} \sin \left(\frac{B \pi V}{V_{\pi}}\right)\right]
$$

where $V$ represents the input voltage and $V_{\pi}$ is the $\pi$-shift voltage assumed to be the same for three MZMs without loss of generality.

In this paper, we choose a two-tone input voltage, comprising two closely spaced frequencies, $f_{1}$ and $f_{2}$.

$$
V=V_{0}\left(\sin \left(2 \pi f_{1} t\right)+\sin \left(2 \pi f_{2} t\right)\right)
$$

where $V_{0}$ is the amplitude.

In this paper, we adopt SFDR to measure the value of the dynamic range. To evaluate the SFDR numerically, we need to apply the modulator in a definite optical link as shown in Figure 3. And Table 1 gives the link parameters. We use the typical values of these parameters to calculate the SFDR value of the optical link $[9,10]$.

SFDR is defined as the ratio of the power of the maximally allowed signal to the power of its accompanying noise when the IMD power equates to the noise power [9]. In the optical link in Table 1, the noise floor is $-147.01 \mathrm{dBm} / \mathrm{Hz}$ including the relative intensity noise of the laser, the shot noise and the thermal noise. According to the definition of SFDR, the powers of the signal and IMD components need to be calculated

\begin{tabular}{ccc}
\hline Quantity & Symbol & Value \\
\hline Laser Power & $P_{L}$ & $0.01 \mathrm{~W}$ \\
\hline Laser Noise & $\mathrm{RIN}$ & $-145 \mathrm{~dB} / \mathrm{Hz}$ \\
\hline Total Optical Loss & $L_{0}$ & $-10 \mathrm{~dB}$ \\
\hline Modulator Sensitivity & $V_{\pi}$ & $5 \mathrm{~V}$ \\
\hline Modulator Impedance & $R_{M}$ & $50 \Omega$ \\
\hline Detector Responsivity & $\eta$ & $0.7 \mathrm{~A} / \mathrm{W}$ \\
\hline Detector Load & $R_{D}$ & $50 \Omega$ \\
\hline Max Current & $P_{L} L_{0} \eta$ & $7 \mathrm{~mA}$ \\
\hline
\end{tabular}

TABLE 1 Link Parameters Used to Evaluate SFDR.

numerically to measure SFDR. As the same as most research $[8,13]$, only IMD3 is considered to calculate the IMD power for the reason that the powers of higher order IMD products are much weaker compared with IMD3. In order to calculate the powers of the fundamental signal and IMD, we need to analyse the coefficients of signal and IMD3 components when the input voltage is in agreement with Eq. (4). Here, we expand Eq. (3) into a Taylor series with respect to $V$ as in Eq. (5).

$$
\begin{aligned}
T=\frac{1}{2} & \left\{1+\frac{1}{A+1}\left[(A-B) \frac{\pi V}{V_{\pi}}-\frac{1}{6}\left(A-B^{3}\right)\right.\right. \\
& \left.\left.\times\left(\frac{\pi V}{V_{\pi}}\right)^{3}+\frac{1}{120}\left(A-B^{5}\right)\left(\frac{\pi V}{V_{\pi}}\right)^{5}+\cdots\right]\right\}
\end{aligned}
$$

As shown in Eq. (5), Eq. (3) is only expanded to the fifth order term since the coefficients of higher order terms are very small and can be neglected. If we introduce Eq. (4) into Eq. (5), we can find the fundamental signal component exists in all order terms except the constant part. Moreover, the IMD3 is generated not only in the third order term but also in the fifth order term. To calculate the powers of the fundamental signal and IMD, we give the coefficients of the fundamental signal in the frequency of $f_{1}$ and IMD3 product in the frequency of $2 f_{1}-f_{2}$ respectively after the introduction of Eq. (4) into Eq. (5).

$$
\begin{aligned}
& C_{f_{1}}=\frac{1}{2(A+1)}[-(A-B) \frac{\pi V_{0}}{V_{\pi}}+\frac{3}{8}\left(A-B^{3}\right) \\
&\left.\times\left(\frac{\pi V_{0}}{V_{\pi}}\right)^{3}-\frac{5}{96}\left(A-B^{5}\right)\left(\frac{\pi V_{0}}{V_{\pi}}\right)^{5}\right] \\
& C_{2 f_{1}-f_{2}}=\frac{1}{2(A+1)}\left[\frac{1}{8}\left(A-B^{3}\right)\left(\frac{\pi V_{0}}{V_{\pi}}\right)^{3}\right. \\
&\left.-\frac{1}{96}\left(A-B^{5}\right)\left(\frac{\pi V_{0}}{V_{\pi}}\right)^{5}\right]
\end{aligned}
$$

where $T_{f}=\frac{1}{\left|f_{1}-f_{2}\right|} \cdot C_{f_{1}}$ and $C_{2 f_{1}-f_{2}}$ represent the coefficients of the fundamental signal in the frequency of $f_{1}$ and the IMD3 product in the frequency of $2 f_{1}-f_{2}$ respectively.

In Eq. (7), it should be noted that most research focuses only on IMD3 in the third order term and then comes to the conclusion that $A$ should be the cubic of $B[8,13]$. More rigid control needs to be applied to the modulator if IMD3 generated in the fifth order term is also taken into account. In the paper, we analyse IMD3 in the third and fifth order terms at the same time and explain how to suppress IMD3 in both terms. 
With the coefficients given, the powers of the fundamental signal and IMD are expressed in Eqs. (8) and (9). The detailed explanations about how to obtain Eqs. (8) and (9) are given in Appendix A.

$$
\begin{gathered}
P_{\text {sig }}=\frac{R_{D}}{2}\left(P_{L} L_{0} \eta C_{f_{1}}\right)^{2} \\
P_{I M D}=\frac{R_{D}}{2}\left(P_{L} L_{0} \eta C_{2 f_{1}-f_{2}}\right)^{2}
\end{gathered}
$$

Furthermore, we can obtain $P_{\text {sig }}$ and $P_{I M D}$ as functions of the input signal power after we deduce the values of $A$ and $B$. Additionally, Appendix $B$ gives the method to deduce the values of $A$ and $B$.

\section{PERFORMANCE AND ANALYSIS}

As mentioned above, we use SFDR to measure the linearity. There will be no ambiguity if we define SFDR as the distance in $\mathrm{dB}$ from the signal to the IMD where the IMD power equals the noise power at the smallest input level [9]. Since the SFDR value is related to the bandwidth of noise and the unit of SFDR is $\mathrm{dB} \cdot \mathrm{Hz}^{(p-1) / p}$ where $\mathrm{p}$ is the dominant order term of IMD3 sources, the unit is different when the dominant IMD3 is generated in different order terms [21]. In the paper, we assume the noise bandwidth is $1 \mathrm{~Hz}$ to avoid the difference in units of SFDR in different cases, which can help compare the performances in different circumstances clearly. And we will demonstrate that the property of the proposed structure is determined by its transfer function (Eq. (3)), therefore, the assumption of bandwidth only affects the values of noise and SFDR instead of the conclusion.

If the bandwidth of noise is $1 \mathrm{~Hz}$, the noise in the selected optical link is $-147.01 \mathrm{dBm}$. The optimum value of $B$ is 2.62 with the optical power penalty considered [9]. According to the Appendix $\mathrm{B}$, the computing result of $A$ is 17.7890 when the value of $B$ is 2.62. The exact cubic value of $B$ is 17.9847 , thus the computing value of $A$ is slightly off the exact cubic of $B$, which is in agreement with previous studies [9]. Figure 4 illustrates the powers of signal and IMD components when the modulator works at its optimum working point. We can find that the SFDR is as high as $104.12 \mathrm{~dB}$ in the case. Figure 4 also shows the performance when the optical power splitting ratio is the exact cubic of the RF voltage splitting ratio as in most research on DPMZM $[8,9,13]$. In this case, $A$ is 17.9847 and SFDR is $100.62 \mathrm{~dB}$. The SFDR when $A$ is 17.7890 is $3.50 \mathrm{~dB}$ larger than that when $A$ is 17.9847 .

To demonstrate the conclusion is not related to the bandwidth of noise, we also analyse the proposed modulator's performance when the bandwidth is $10 \mathrm{kHz}$ as shown in Figure 5 . In this case, the value of the noise and matched value of $A$ is $-107.01 \mathrm{dBm}$ and 16.7813 respectively. The matched value of $A$ is still off the exact cubic of $B$. Moreover, the SFDR is $71.93 \mathrm{~dB}$ when $A$ is $16.7813,3.31 \mathrm{~dB}$ larger than the SFDR when $A$ is 17.9847 .

In Figures 4 and 5, the differences of SFDR when $A$ is off the exact cubic of and exact cubic of $B$ are mainly caused by the IMD powers as the curves of fundamental signal's powers in

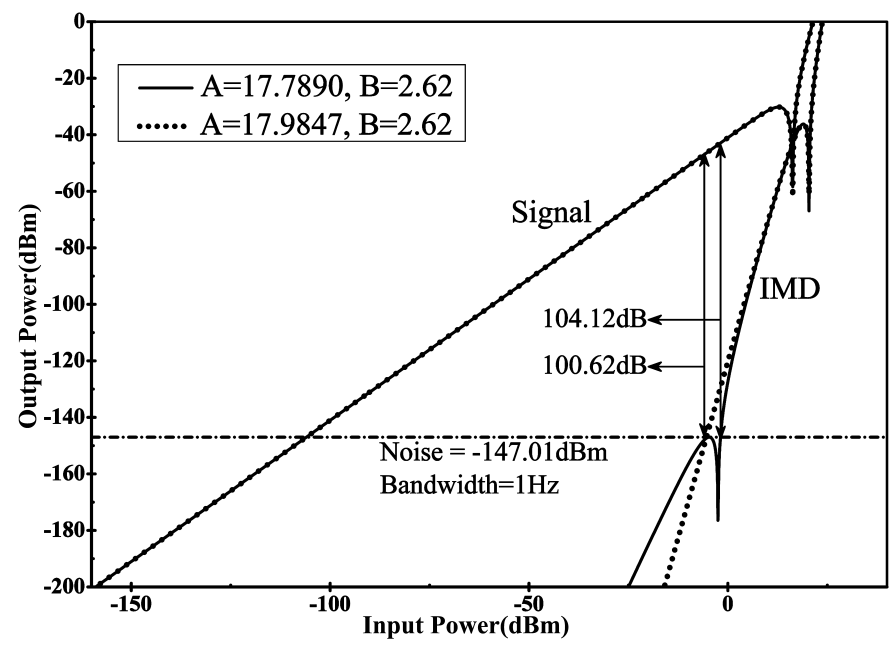

FIG. 4 Output fundamental signal power, IMD power as a function of the input signal power for a fibre-optic link (Table 1 parameters) when i) $A$ is off the exact cubic of $B$, ii) $A$ is the exact cubic of $B$.

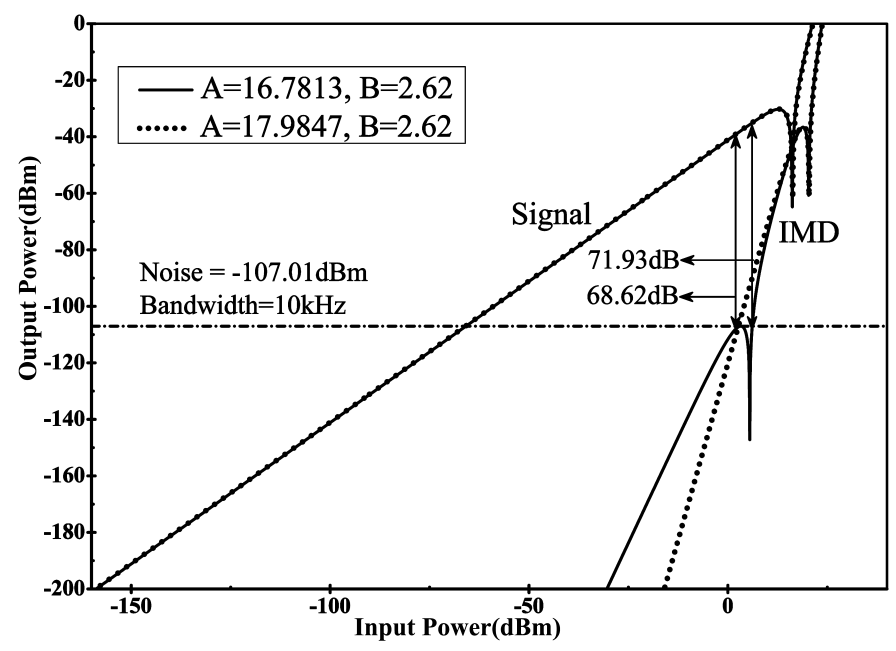

FIC. 5 Output fundamental signal power, IMD power as a function of the input signal power for a fibre-optic link (Table 1 parameters) when i) $A$ is off the exact cubic of $B$, ii) $A$ is the exact cubic of $B$.

two circumstances nearly coincide. In both figures, a hump exists in the curve of the IMD's power if $A$ is off the exact cubic of $B$ while there is no hump if $A$ is 17.9847 . The top of the hump represents the local maximum value of IMD's power which is just below the noise level. If the local maximum value exceeds the noise level, the SFDR decreases sharply according to its definition. Moreover, when $A$ is off the cubic value of $B$, the curve of IMD is steeper than the curve when $A$ is 17.9847. The slope of the IMD's curve when $A$ is 17.9847 is 5.00 , meaning that the residual IMD3 is from the fifth order term in Eq. (5) and IMD3 in the third order term is totally prohibited. However, when $A$ is 17.7890 or 16.7813 , the slope of the IMD's curve is larger than 5.00, indicating that IMD3 in the fifth order term is partly cancelled. Further calculation demonstrates that almost all IMD3 in the third order term and part of IMD3 in the fifth order term is prohibited and the suppression of IMD3 from the fifth order term is much larger than the residual IMD3 from the third order term. It can be concluded that the SFDR can obtain further improvement if the optical power splitting ratio is off the exact cubic of the RF voltage splitting ratio. 


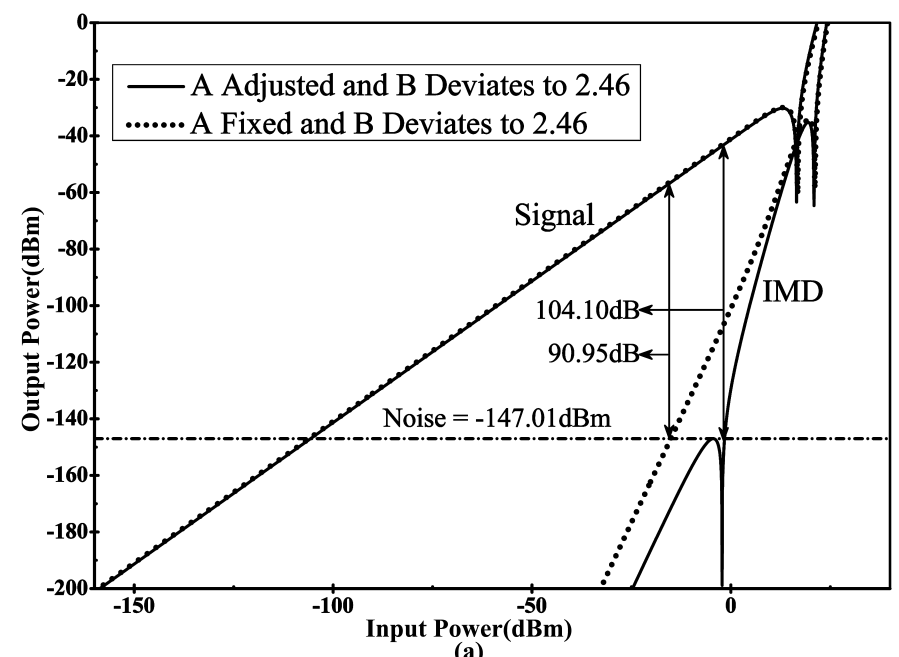

(a)

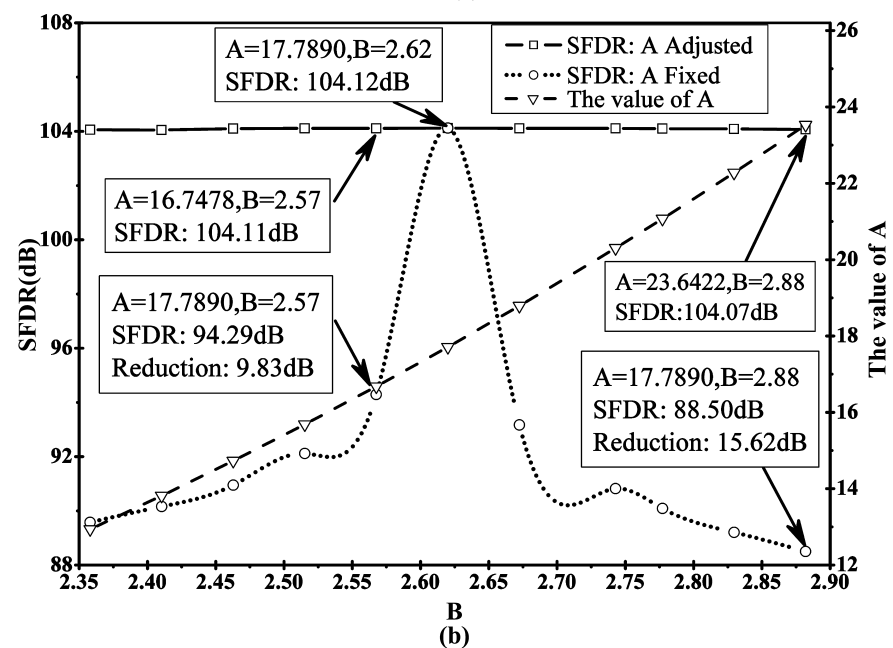

FIG. 6 (a) Output fundamental signal power, IMD power as a function of the input signal power for a fibre-optic link (Table 1 parameters) when MMI-DPMZM operates in two conditions: $i)$ the value of $A$ is adjusted to 14.7882 when the value of $B$ deviates to 2.46 , ii) the value of $A$ is fixed at 17.7890 when the value of $B$ deviates to 2.46 . (b) With $B$ deviating in the range of $\pm 10 \%$, the values of SFDR in two conditions: $i$ ) $A$ is adjusted proportionately, ii) $A$ is fixed at 17.7890 . The matched $A$ for different values of $B$ is also shown in the figure.

As mentioned in Section 2, the value of $A$ is chosen to be the exact cubic of $B$ in previous papers on DPMZM $[8,9,13]$. The SFDR performance of conventional DPMZM is the same as that of the proposed structure when $A$ is 17.9847. However, the SFDR of MMI-DPMZM can be further improved about $3 \mathrm{~dB}$ if $A$ is off the exact cubic of $B$. Therefore, we can conclude that the peak SFDR of the proposed structure is about $3 \mathrm{~dB}$ larger than that of conventional DPMZM in previous papers $[8,9,13]$. Additionally, the SFDR value is smaller than the previous research $[9,13,14]$ because the values of link parameters as shown in Table 1 are different from the values in previous research. In this paper, the values of the link parameters are much more available for modern technologies.

For traditional DPMZM, the high SFDR can be achieved only when the splitting ratios of optical power and RF voltage match each other exactly. If anyone deviates from the matched value, the linearity will deteriorate sharply [9]. We also study the proposed structure's performance when the RF splitting ratio deviates from its optimum value and Figure 6(a) shows the results. In the proposed structure, $A$, the optical power splitting ratio can be adjusted through $\mathrm{MMI}_{a}$. Figure 6(a) illustrates the SFDR values with and without the value of $A$ adjusted when the value of $B$ deviates to 2.46. If $A$ is fixed at 17.7890, the SFDR falls to $90.95 \mathrm{~dB}$. However, when $A$ is adjusted to the matched value of 14.7882, the SFDR can recover to $104.10 \mathrm{~dB}$, only $0.02 \mathrm{~dB}$ smaller than the peak value. For further demonstration, Figure 6(b) compares the variations of the SFDR value when the deviation of $B$ is within the range of $\pm 10 \%$ in two cases: (i) $A$ is fixed and (ii) $A$ is adjusted. In the figure, we see that the deviation of $B$ leads to a significant drop of the SFDR value as large as $15.62 \mathrm{~dB}$ if we fix the value of $A$. However, the SFDR can be maintained above $104.00 \mathrm{~dB}$ when we make some compensation to the deterioration through adjusting $A$. Figure 6(b) also shows the matched value of $A$ when $B$ changes from 2.36 to 2.88 . If $B$ deviates to 2.88 , the value of $A$ has to be increased to as high as 23.6422, which can be achieved through the adjustment of the voltage $V_{a}$ [18]. The adjustment of $\mathrm{MMI}_{a}$ can change the value of $A$, ensuring the modulator keep stable SFDR in a large operating range, which greatly increases the modulator's design flexibility, manufacturing tolerance and wide application. Here, it can be demonstrated the SFDR performance of conventional DPMZM is the same as that of MMI-DPMZM when the value of $A$ is fixed. However, the proposed structure has wider operating range and is much more tolerant compared with the conventional DPMZM due to the adjustment of the MMI coupler. Though some novel Y-splitters can also achieve precise splitting [22, 23], MMI couplers are more robust to manufacturing deviation and environmental changes $[15,19]$. Moreover, the $3 \mathrm{~dB}$ MMI couplers also offer the relative phases which replace the DC bias circuits on $\mathrm{MZM}_{b}$ and $\mathrm{MZM}_{c}$.

\section{DISCUSSION}

It can be concluded from Figure 6(b) that the proposed structure can create an extremely stable dynamic range whose reduction is not more than $0.10 \mathrm{~dB}$ with the reconfigurable MMI coupler adjusted. However, in above analysis, the precision of the reconfigurable MMI coupler requires $4^{\text {th }}$-decimal order and no imbalance exists for the $3 \mathrm{~dB}$ MMI couplers, which may lead to complex control circuits conversely. In recent decades, numerous MMI structures have been proposed, including adjustable types [18, 19] and fixed ones [16, 17]. For the reconfigurable types, the precision of $2^{\text {nd }}$-decimal order of the optical power splitting ratio can be obtained [19]. The imbalance of $3 \mathrm{~dB}$ MMI couplers caused by the manufacturing errors can be improved. Uniformity (UF) is often used to measure the imbalance of $3 \mathrm{~dB}$ MMI couplers, which is calculated as $-10 \lg \left(P_{\min } / P_{\max }\right)$, where $P_{\max }$ and $P_{\min }$ are the maximum and minimum powers of the MMI output waveguides respectively. In ideal circumstance, UF is $0 \mathrm{~dB}$. For some modern MMI couplers, UF can be reduced to below $1 \mathrm{~dB}[16,17]$. To analyse the performance more generally, we assume that the value of UF is $1 \mathrm{~dB}$. In this case, the $3 \mathrm{~dB}$ MMI couplers split the input light in a power ratio of $1.115: 0.885$. We can get the transfer function of the proposed structure as in Eq. (10). 


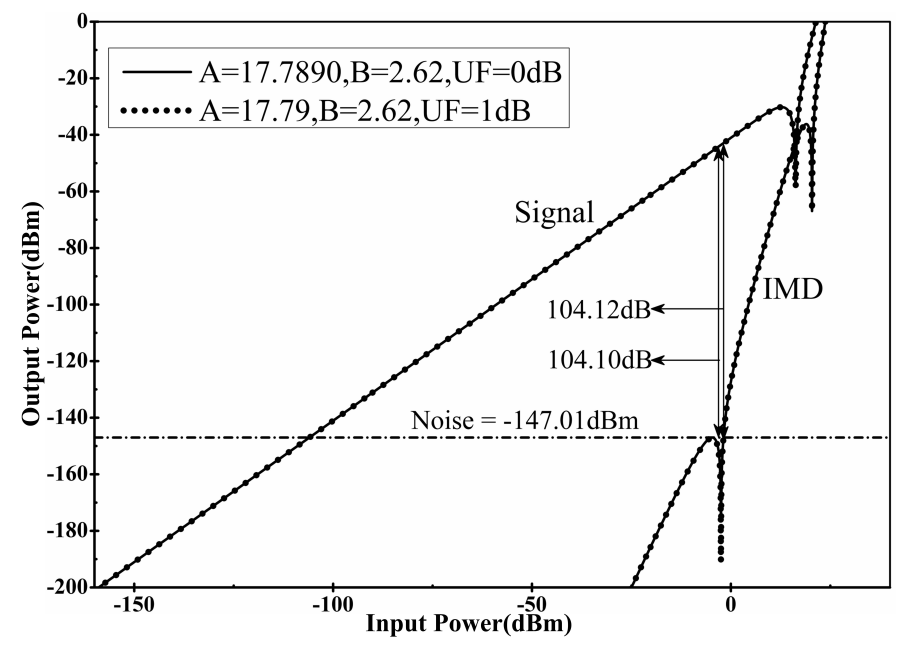

FIG. 7 Output fundamental signal power, IMD power as a function of the input signal power for a fibre-optic link (Table 1 parameters) when MMI-DPMZM operates in two conditions: $\mathrm{i})$ the ideal condition $(A=17.7890, B=2.62, U F=0 \mathrm{~dB})$, ii) the actual condition $(A=17.79, B=2.62, U F=1 d B)$.

$$
\begin{aligned}
T=\frac{1}{2} & {\left[1.0033+\frac{0.9968}{A+1}\left(A \sin \left(\frac{\pi V}{V_{\pi}}\right)\right.\right.} \\
& \left.\left.-\frac{1}{A+1} \sin \left(\frac{B \pi V}{V_{\pi}}\right)\right)\right]
\end{aligned}
$$

To ensure the hump not above the noise level, we should round the optical power splitting ratio to the nearest value in $2^{\text {nd }}$-decimal order greater than or equal to the ideal value in $4^{\text {th }}$-decimal order. Figure 7 compares the performances of MMI-DPMZM in ideal circumstance and in the actual condition. It can be found that the dynamic range is $104.10 \mathrm{~dB}$ when $A=17.79$ and $U F=1 \mathrm{~dB}$, just $0.02 \mathrm{~dB}$ smaller than that in ideal circumstance. The difference of SFDR in two circumstances is so slight that the curves nearly coincide in Figure 7.

\section{CONCLUSION}

In this paper, a modified DPMZM structure with MMI couplers functioning as optical splitters is proposed and analysed. We find that the structure has a dynamic range as large as $104.12 \mathrm{~dB}$. More importantly, the flexibility of the tunable MMI coupler increases the modulator's tolerance for underlying shift of the operating point. When the RF voltage splitting ratio deviates from its optimum value, the structure can still maintain the high dynamic range above $104.00 \mathrm{~dB}$ through the adjustment of the reconfigurable MMI coupler. Moreover, detailed analysis demonstrates that the proposed structure has good tolerance to MMI couplers' manufacturing errors. The dynamic range can reach $104.10 \mathrm{~dB}$ when the precision of the reconfigurable MMI coupler is $2^{\text {nd }}$-decimal order and the uniformity of the $3 \mathrm{~dB}$ MMI couplers is $1 \mathrm{~dB}$. Additionally, the MMI coupler is insensitive to the environmental factors and can be easily integrated.

\section{ACKNOWLEDGEMENT}

This work was supported by the National Nature Science Foundation of China grant No. 60902038, the 111 project under Grant No.B08038 and the Fundamental Research Funds for the Central Universities No.K50511010019.

\section{APPENDIX}

\section{A Powers of Fundamental Signal and IMD}

According to the definition of SFDR, it is necessary to calculate the powers of the fundamental signal and IMD. Since IMD3 predominates in IMD products, only IMD3 is considered to calculate the power of IMD. Therefore, the powers of the fundamental signal and IMD can be expressed as follows $[9,10]$.

$$
\begin{gathered}
P_{\text {sig }}=\frac{R_{D}}{2}\left[P_{L} L_{0} \eta \cdot \frac{2}{T_{f}} \int_{0}^{T_{f}} T \cdot \exp \left(-2 \pi f_{1} t\right) d t\right]^{2} \\
P_{I M D}=\frac{R_{D}}{2}\left[P_{L} L_{0} \eta \cdot \frac{2}{T_{f}} \int_{0}^{T_{f}} T \cdot \exp \left(-2 \pi\left(2 f_{1}-f_{2}\right) t\right) d t\right]^{2}
\end{gathered}
$$

where $T$ represents the transfer function of the proposed structure.

Eqs. (11) and (12) consist of Fourier transformations of the transfer function. According to the definition of the Fourier decomposition, the coefficients of the fundamental signal in the frequency of $f_{1}$ and the IMD3 product in the frequency of $2 f_{1}-f_{2}$ can be obtained through Eqs. (13) and (14).

$$
\begin{gathered}
C_{f_{1}}=\frac{2}{T_{f}} \int_{0}^{T_{f}} T \cdot \exp \left(-2 \pi f_{1} t\right) d t \\
C_{2 f_{1}-f_{2}}=\frac{2}{T_{f}} \int_{0}^{T_{f}} T \cdot \exp \left(-2 \pi\left(2 f_{1}-f_{2}\right) t\right) d t
\end{gathered}
$$

Then, Eqs. (11) and (12) can be simplified with Eqs. (13) and (14). The simplified equations are given as follows.

$$
\begin{gathered}
P_{\text {sig }}=\frac{R_{D}}{2}\left(P_{L} L_{0} \eta C_{f_{1}}\right)^{2} \\
P_{I M D}=\frac{R_{D}}{2}\left(P_{L} L_{0} \eta C_{2 f_{1}-f_{2}}\right)^{2}
\end{gathered}
$$

\section{B Values of $A$ and $B$}

In Eqs. (15) and (16), the values of $A$ and $B$ are necessary to calculate the powers of the signal and IMD components. The value of $B$ is specified independently with the optical power penalty considered and its optimum value is 2.62 [9]. In order to get the matched value of $A$, we need to analyse the coefficient of the IMD3 product as shown in Eq. (17).

$C_{2 f_{1}-f_{2}}=\frac{1}{2(A+1)}\left[\frac{1}{8}\left(A-B^{3}\right)\left(\frac{\pi V_{0}}{V_{\pi}}\right)^{3}-\frac{1}{96}\left(A-B^{5}\right)\left(\frac{\pi V_{0}}{V_{\pi}}\right)^{5}\right.$ 
Studying the monotonic property of Eq. (17), we can get a local maximum value of the IMD power when the following equation is satisfied.

$$
\pi \frac{V_{0}}{V_{\pi}}=\frac{6}{\sqrt{5}} \sqrt{\frac{A-B^{3}}{A-B^{5}}}
$$

According to the previous study [9], the largest dynamic range occurs when the IMD power at the point that the input voltage satisfies Eq. (18) is just below the noise level. The relationship is described in Eq. (19).

$$
P_{I M D} \leq P_{\text {noise }}
$$

If the noise bandwidth is given, the value of $P_{\text {noise }}$ can be obtained. Then, we can introduce Eqs. (16) and (17) into Eq. (19) to get the optimum value of $A$.

\section{References}

[1] P. T. Dat, A. Bekkali, K. Kazaura, K. Wakamori, and M. Matsumoto, "A universal platform for ubiquitous wireless communications using Radio over FSO system," J. Lightwave Technol. 28, 2258-2267 (2010).

[2] M. Zhu, L. Zhang, J. Wang, L. Cheng, C. Liu, and C. Gee-Kung, "Radio-Over-Fiber access architecture for integrated broadband wireless services," J. Lightwave Technol. 31, 3614-3620 (2013).

[3] C. Chen, C. Zhang, W. Zhang, W. Jin, and K. Qiu, "Scalable and reconfigurable generation of flat optical comb for WDM-based nextgeneration broadband optical access networks," Opt. Commun. 321, 16-22 (2014).

[4] T. Kanesan, W. Pang Ng, Z. Ghassemlooy, and C. Lu, "Investigation of optical modulators in optimized nonlinear compensated LTE ROF system," J. Lightwave Technol. 32, 1944-1950 (2014).

[5] Y. Hsueh, Z. Jia, H. Chien, A. Chowdhury, J. Yu, and G. Chang, "Multiband 60- $\mathrm{CHz}$ wireless over fiber access system with high dispersion tolerance using frequency tripling technique," J. Lightwave Technol. 29, 1105-1111 (2011).

[6] C. J. Hansen, "WiGiG: Multi-gigabit wireless communications in the $60 \mathrm{GHz}$ band," IEEE Wirel. Commun. 18, 6-7 (2011).

[7] G. S. D. Cordon, M. J. Crisp, R. V. Penty, and I. H. White, “High-Order distortion in directly modulated semiconductor lasers in high-loss analog optical links with large RF dynamic range," J. Lightwave Technol. 29, 3577-3586 (2011).

[8] L.M. Johnson, and H. V. Roussell, "Reduction of intermodulation distortion in interferometric optical modulators," Opt. Lett. 13, 928-930 (1988).

[9] W. B. Bridges, and J. H. Schaffner, "Distortion in linearized electrooptic modulators," IEEE Trans. Microwave Theory Tech. 43, 2184-2197, (1995).

[10] A. Prescod, B. B. Dingel, N. Madamopoulos, and R. Madabhushi, "Effect of ring resonator waveguide loss on SFDR performance of highly linear optical modulators under suboctave operation," IEEE Photon. Tech. L. 22, 1297-1299 (2010).
[11] E. H. W. Chan, W. Zhang, and R. A. Minasian, "Photonic RF phase shifter based on optical carrier and RF modulation sidebands amplitude and phase control," J. Lightwave Technol. 30, 3672-3678 (2012).

[12] J. Li, T. Ning, L. Pei, W. Jian, H. You, H. Chen, C. Zhang, C. Li, "Optical single sideband modulation with continously tunable optical carrier-to-sideband ratio by employing a dual-parallel MachZehnder modulator," Acta Phys. Sin. 62 (2013).

[13] S. Li, X. Zheng, H. Zhang, and B. Zhou, "Highly linear Radioover-Fiber system incorporating a single-drive Dual-Parallel MachZehnder modulator," IEEE Photon. Tech. L. 22, 1775-1777 (2010).

[14] Y. Gao, A. Wen, Q. Yu, N. Li, G. Lin, S. Xiang, L. Shang, “Microwave Generation With Photonic Frequency Sextupling Based on Cascaded Modulators," IEEE Photon. Tech. L. 26, 1199-1202 (2014).

[15] D. J. Thomson, Y. Hu, G. T. Reed, and J. M. Fedeli, "Low loss MMI couplers for high performance MZI modulators," IEEE Photon. Tech. L. 22, 1485-1487, (2010).

[16] S. Musa, N. S. Lagali, G. Sengo, G. J. M. Krijnen, and A. Driessen, "Design and fabrication of $1 \times \mathrm{N}$ and $\mathrm{NxN}$ planar waveguide couplers for multimode fiber-based local area networks," in Proceedings of IEEE/LEOS Benelux Chapter 2001 Annual Symposium (IEEE, Twente, 2001).

[17] A. Hosseini, H. Subbaraman, D. Kwong, Y. Zhang, and R. T. Chen, "Optimum access waveguide width for $1 \times \mathrm{N}$ multimode interference couplers on silicon nanomembrande," Opt. Lett. 35, 28642865 (2010).

[18] R. Thapliya, T. Kikuchi, and S. Nakamura, "Tunable power splitter based on an electro-optic multimode interference device," Appl. Optics 46, 4155-4161 (2007).

[19] D. A. May-Arrioja, P. LikamWa, J. J. Sanchez-Mondragon, R. J. Selvas-Aguilar, and I. Torres-Gomez, "A reconfigurable multimode interference splitter for sensing applications," Measurement Science and Technol. 18, 3241-3246 (2007).

[20] M. Bachmann, P. A. Besse, and H. Melchior, "Overlapping-image multimode interference couplers with a reduced number of selfimages for uniform and nonuniform power splitting," Appl. Optics 34, 6898-6910 (1995).

[21] E. I. Ackerman, "Broad-band Linearization of a Mach-Zehnder Electrooptic Modulator," IEEE Trans. Microw. Theory Techniques, 47, 2271-2279 (1999).

[22] Y. Zhang, S. Y. Yang, A. E. Lim, G. Q. Lo, C. Galland, T. B. Jones, and M. Hochberg, "A compact and low loss $Y$-junction for submicron silicon waveguide," Opt. Express 21, 1310-1316 (2013).

[23] A. Rajandekar, and R. Singhal, "Voltage-controlled all-polymer reconfigurable optical power splitter," in Proceedings of IEEE 4th International Conference on Photonics 244-246 (IEEE, Melaka, 2013). 